\title{
Importância do exame oftalmológico na doença de von Hippel-Lindau
}

\section{The importance of the ophthalmological examination in von Hippel-Lindau disease}

Ricardo Evangelista Marrocos de Aragão ${ }^{1}$, lêda Maria Alexandre Barreira ${ }^{2}$, Andreya Ferreira Rodrigues Bezerra ${ }^{3}$ Régia Maria Gondim Ramos ${ }^{4}$, Felipe Bezerra Alves Pereira ${ }^{5}$

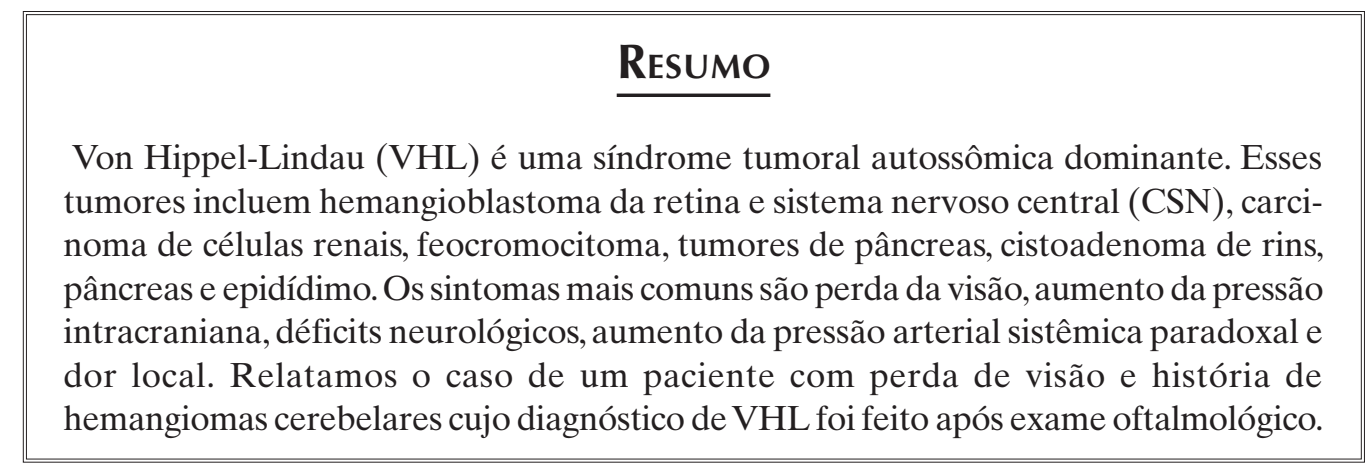

Descritores: Doença de von Hippel-Lindau; Hemangioblastoma; Hamartoma; Feocromocitoma; Hipertensão intracraniana; Relatos de casos

\footnotetext{
'Doutor em Medicina pela Universidade de Regensburg - Alemanha; Especialista em Retina e Vítreo pela Universidade de Regensburg - Alemanha; Preceptor da Residência Médica de Retina e Vítreo do Departamento de Oftalmologia do Hospital Universitário Walter Cantídio da Universidade Federal do Ceará - UFC - Fortaleza (CE), Brasil;

${ }^{2}$ Especialista em Retina e Vítreo pela Universidade Federal de São Paulo - UNIFESP - São Paulo (SP), Brasil;

${ }^{3}$ Residente do terceiro ano do Serviço de Oftalmologia do Hospital Universitário Walter Cantídio da Universidade Federal do Ceará UFC - Fortaleza (CE), Brasil;

${ }^{4}$ Residente do terceiro ano do Serviço de Oftalmologia do Hospital Universitário Walter Cantídio da Universidade Federal do Ceará UFC - Fortaleza (CE), Brasil;

${ }^{5}$ Ex-residente do Serviço de Oftalmologia do Hospital Universitário Walter Cantídio da Universidade Federal do Ceará - UFC Fortaleza (CE), Brasil.
}

Instituição: Serviço de Oftalmologia do Hospital Universitário Walter Cantídio da Universidade Federal do Ceará - UFC - Fortaleza (CE), Brasil

Recebido para publicação em: 9/4/2009 - Aceito para publicação em 22/9/2009 


\section{INTRODUÇÃO}

A doença de von Hippel-Lindau (VHL), ou angiomatose cerebelorretiniana familiar, é uma facomatose autossômica dominante, com penetrância incompleta e expressividade retardada ${ }^{(1)}$ caracterizada por aparecimento de hemangioblastoma da retina, do cerebelo e/ou medula espinhal, carcinoma de células renais, tumor de pâncreas, tumores do saco endolinfático, bem como cistos e cistoadenoma dos rins, pâncreas e epidídimo ${ }^{(2)}$. Diferindo de outras facomatoses, as manifestações cutâneas são raras em VHL. Outras manifestações de VHL que podem chamar atenção do oftalmologista incluem retinopatia hipertensiva devido a feocromocitoma, hamartoma vascular da retina, síndrome quiasmática, defeito pupilar aferente devido a hemangioblastoma do nervo óptico (NO) e papiledema por lesões do SNC ou do nervo óptico ${ }^{(3)}$.

$\mathrm{O}$ hemangioma capilar da retina (HCR) é a manifestação mais precoce da doença de VHL e usualmente não está presente ao nascimento. Existe uma correlação positiva entre o aumento da idade e o risco de desenvolvimento de HCR na VHL. O HCR pode ser um tumor único e algumas vezes é a única manifestação da patologia, mas os tumores são mais frequentemente múltiplos, ocorrendo em $45 \%$ a $60 \%$ dos pacientes com VHL. A localização preferencial do tumor é a periferia temporal da retina, sendo facilmente reconhecido por sua aparência avermelhada, globulosa, com uma artéria nutridora e uma veia tortuosa que drena o tumor ${ }^{(4)}$.Apesar da sua natureza benigna e clássico crescimento lento, o HCR pode causar complicações como descolamento total da retina, catarata e phthisis bulbi nos estágios finais ${ }^{(5)}$. O HCR permanece como a maior causa de morbidade visual e algumas vezes cegueira nos pacientes com VHL. A detecção precoce e tratamento podem alterar o prognóstico visual ${ }^{(4)}$.

No presente artigo, relatamos o caso de um paciente portador de VHL com história prévia de cirurgias neurológicas para remoção de hemongioblastoma cerebelar, perda da visão com phthisis bulbi no olho direito (OD) e presença de HCR no olho esquerdo(OE), no qual foi realizado tratamento com laser.

\section{Relato de Caso}

Paciente masculino, 29 anos, branco, procurou o ambulatório com queixa de baixa acuidade visual acompanhada de discreta dor em região orbitária direita. $\mathrm{Pa}$ ciente tinha história pregressa de três cirurgias neurológicas para ressecção de hemangioblastoma cerebelar (diagnóstico proposto antes das cirugias por exames de imagem, tomografia computadorizada de SNC, e confirmado com os exames anátomo-patológicos) e cistos de epidídimo. História familiar negativa.

Ao exame oftalmológico, o paciente apresentava acuidade visual (AV) de ausência de percepção luminosa no olho direito (OD) e 20/20 no olho esquerdo (OE). A biomicroscopia mostrou desorganização das estruturas do segmento anterior do OD e OE sem alterações. À oftalmoscopia binocular indireta revelou descolamento total de retina no OD e no OE um HCR de localização temporal inferior. A ecografia ocular mostrou descolamento de retina associado a espessamento de esclera e desorganização das estruturas (Phitisis bulbi) no OD. A angiografia fluoresceínica (AF) no OE mostrou lesão hiperfluorescente temporal inferior, com vasos aferente e eferente característicos do HCR (Figura 1).

O paciente foi submetido, no OE, à fotocoagulação com laser de diodo verde na lesão e vasos nutridores. Após 20 dias, realizou-se nova AF, que mostrou regressão da lesão (Figura 2).

\section{Discussão}

Os hemangiomas da retina que ocorrem esporadicamente ou em associação com VHL são clinicamente indistinguíveis. O diagnóstico de VHL se baseia em três elementos, que incluem: hemangioma (retina/SNC), lesões viscerais e história familiar de lesões similares. Se história familiar de VHL está presente, apenas uma lesão é requerida. Com história familiar negativa, presença de pelo menos dois hemangiomas ou um hemangioma e uma lesão visceral são critérios para o diagnóstico de $\mathrm{VHL}^{(6)}$. Tumores assintomáticos podem ser detectados tão precocemente quanto na primeira década de vida ou tão tardiamente quanto na nona década em uma retina previamente normal. Embora o HCR seja um hamartoma, usualmente não está presente congenitamente. Comumente, hemangiomas da retina são detectados em pacientes entre 25 e 30 anos, quando eles têm crescido suficientemente para serem diagnosticados clinicamente no exame de rotina ${ }^{(7)}$. A história natural do HCR consiste de progressão com o tempo. Entretanto, alguns hemangiomas permanecem estáveis e alguns podem até regredir espontaneamente. $\mathrm{O} H C R$ pode exsudar, podendo levar a perda visual por maculopatia $^{(7)}$ e descolamento de retina seroso ou tracional $^{(8)}$. Embora rara, neovascularização de retina e íris pode ocorrer, potencialmente evoluindo para 

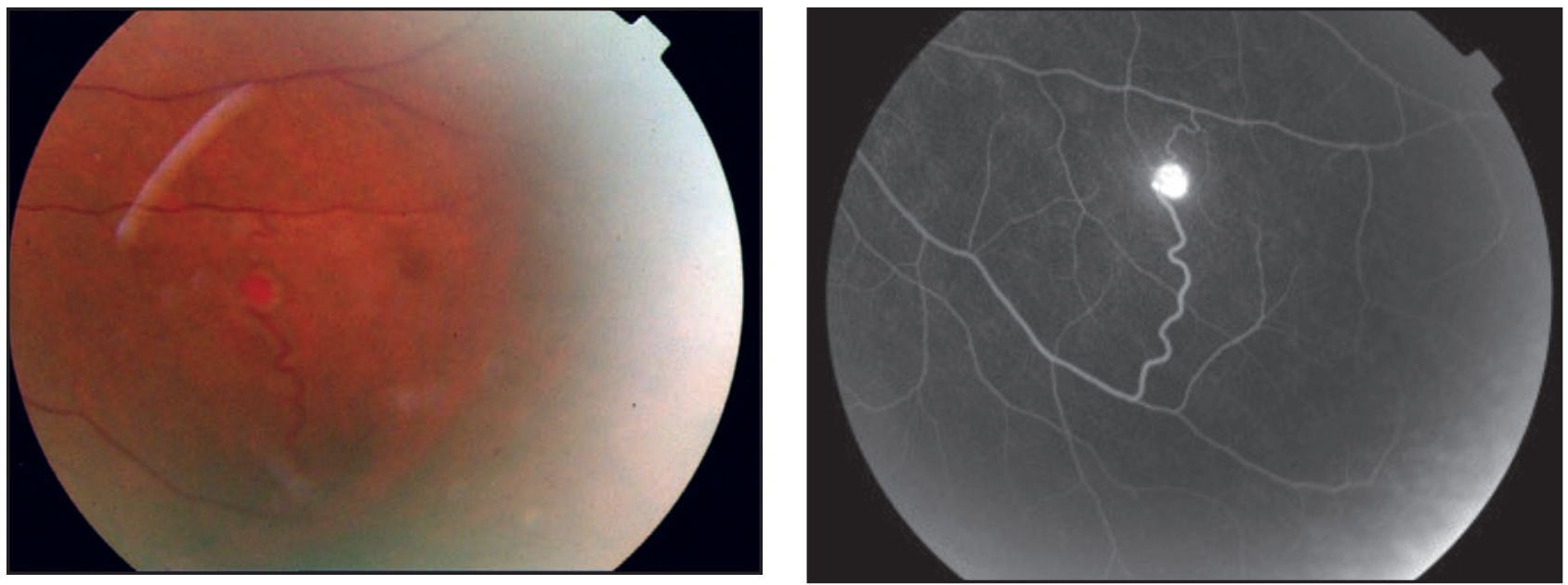

Figura 1: Hemangioblastoma em retina temporal e angiofluoresceinografia com hiperfluorescência
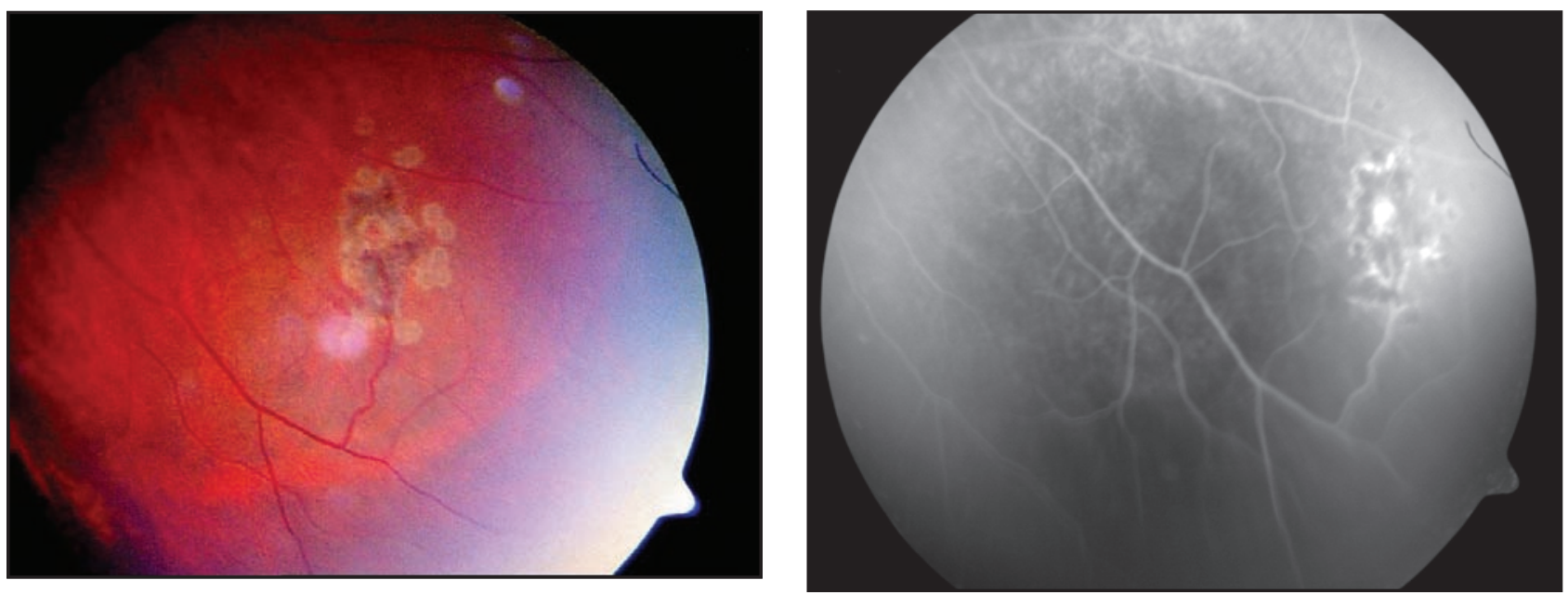

Figura 2: Retinografia simples e angiofluoresceinografia após fotocoagulação a laser do tumor

glaucoma secundário e phtisis bulbi ${ }^{(7)}$.

A decisão de tratar ou monitorar o tumor depende do tamanho e localização da lesão. Tratamento mais agressivo deve ser instituído para lesões periféricas. Tumores periféricos menores que $4,5 \mathrm{~mm}$ de diâmetro e $1 \mathrm{~mm}$ de espessura são tratados com fotocoagulação a laser. Em tumores maiores, usa-se crioterapia como método adjuvante.

Como o hemangioma capilar de retina pode ser a única manifestação da doença de von Hippel-Lindau, pacientes com esse achado devem ser investigados para tal patologia através de ressonância nuclear magnética ou tomografia computadorizada de SNC, coluna cervical e abdome ${ }^{(7)}$.

No caso relatado, o oftalmologista teve um papel essencial no diagnóstico de VHL, a despeito do paciente ter história pregressa de cirurgia neurológica para exérese de hemangioblastoma cerebelar e perda visual no OD. Foi instituído o tratamento com fotocoagulação a laser no OE com regressão da lesão.

\section{Abstract}

Von Hippel-Lindau (VHL) disease is an autossomical, dominant inherited tumour syndrom. These tumours may include haemangioblastoma in the retina and central nervous system (CNS), renal cell carcinoma, phaeochromocytoma, islet cell tumours of the pancreas, cystadenoma in the kidney, pancreas, and epididymis. The most common symptoms include: loss of vision, raised intracranial pressure, neurological deficits, paroxysmal raised blood pressure and local pain. We report herein a 29-year-old man with visual loss and cerebellar haemangioblastoma that despite neurological manifestations the diagnosis of VHL was established after the ophthalmological examanination. 
Keywords: von Hippel-Lindau disease; Hemangioblastoma; Hamartoma; Pheochromocytoma; Intracranial hypertension; Case reports

\section{REFERÊNCIAS}

1. Tumours of the uvea and retina. In: Kanski, Jack J. Clinical ophthalmology. A systemic approach. Oxford;1993.

2. Hes FJ, van der Luijt RB, Lips CJ. Clinical management of Von Hippel-Lindau (VHL) disease. Neth J Med. 2001;59(5):225-34. Review.

3. Hinz BJ, Schachat AP. Capillary hemangioma of the retina and von Hippel-Lindau disease. In: Ryan SJ, editor. Retina. New York: Mosby; 2006.

4. Dollfus H, Massin P, Taupin P, Nemeth C, Amara S, Giraud S, et al. Retinal hemangioblastoma in von Hippel-Lindau disease: A clinical and molecular study. Invest Ophthalmol Vis Sci. 2002; 43(9):3067-74.

5. Shields JA, Shields CL. Tumors of the retina and optic disc. In: Regillo CD, Brown GC, Flynn HW, editor. Vitreoretinal disease. The essentials. New York: Thieme; 1999.
6. Singh AD, Nouri M, Shields CL, Shields JA, Smith AF. Retinal capillary hemangioma. A comparison of sporadic cases and cases associated with von Hippel-Lindal disease. Ophthalmology. 2001; 108(10):1907-11.

7. Aumiller MS. Juxtapapillary hemangioma: a case report and review of clinical features and management of von HippelLindau disease. Optometry. 2005;76(8):442-9.

8. Barreira IMA, Bordon AF, Tavano V, Uno F, Guia T. Hemangioma capilar da retina associado a descolamento de retina. Relato de caso. Arq Bras Oftalmol. 1999;62(3):320-4.

\section{Endereço para correspondência:}

Ricardo E Marrocos de Aragão

Rua Osvaldo Cruz, no 2335

CEP 60125-151 - Fortaleza - CE

Tel: (85)9137-9313

E-mail: ricardomarrocos@yahoo.com 